\title{
Perioperative Use of Flurbiprofen Axetil on Renal Function after Transurethral Prostatectomy: A Prospective Randomized Controlled Study
}

\author{
Dong Wang $\mathbb{D},{ }^{1}$ Wenxiu Xie $\mathbb{D}^{1},{ }^{1}$ Bo Li $\mathbb{D},{ }^{2}$ Yufan Zhao $\mathbb{D}^{1},{ }^{1}$ Xing Liu $\mathbb{D},{ }^{1}$ Yufeng Zhang $\mathbb{D},{ }^{1}$ \\ Yongzhong Tang $\oplus^{1,3}$ and Xinlin Yin $\oplus^{1,4}$ \\ ${ }^{1}$ Department of Anesthesiology, The Third Xiangya Hospital, Central South University, Changsha, 410013 Hunan, China \\ ${ }^{2}$ Surgery Center, The Third Xiangya Hospital, Central South University, Changsha, 410013 Hunan, China \\ ${ }^{3}$ Hunan Key Laboratory of Brain Homeostasis, China \\ ${ }^{4}$ Hunan Key Laboratory of Medical Information Research, China
}

Correspondence should be addressed to Xinlin Yin; yxl_email@126.com

Received 30 January 2021; Revised 3 February 2021; Accepted 15 February 2021; Published 27 February 2021

Academic Editor: Tingting Hong

Copyright (C) 2021 Dong Wang et al. This is an open access article distributed under the Creative Commons Attribution License, which permits unrestricted use, distribution, and reproduction in any medium, provided the original work is properly cited.

\begin{abstract}
Objective. To explore the role of low-dose flurbiprofen axetil in perioperative renal protection. Methods. A total of 83 patients who underwent transurethral resection of the prostate (TURP) between August 2020 and November 2020 at the Third Xiangya Hospital of Central South University were selected, aged 60-85 years old, American Society of Anesthesia (ASA) physical status classes $1-3$, BMI $18-30 \mathrm{~kg} / \mathrm{m}^{2}$, randomly divided into the experimental group (group F, $n=42$ ) and control group (group C, $n=41) .10$ minutes before the operation, group F was injected with $100 \mathrm{mg}(10 \mathrm{~mL})$ flurbiprofen axetil, and group $\mathrm{C}$ was injected with $10 \mathrm{~mL} 0.9 \%$ saline, comparing the incidence of acute kidney injury (AKI) and changes in glomerular filtration rate (eGFR) between the two groups. Cystatin-C, Neutrophil Gelatinase-associated Lipocalin (NGAL), IL-6, and CRP were compared between the two groups at 4 time points (before surgery, 6 hours, 24 hours, and 48 hours after surgery). Results. A total of 80 cases were enrolled, 40 in group F and 40 in group C. There was no significant difference in baseline between the two groups $(P>0.05)$. The NGAL of group $\mathrm{F}$ was significantly lower than group $\mathrm{C}$ at 6 hours after the operation $(367.99 \pm 311.83$ vs. $243.02 \pm 151.73, P=0.026)$, but there was no significant difference in NGAL and Cystatin-C at other time points $(P>0.05)$. And there was no significant difference in the incidence of postoperative AKI between the two groups $(0 \%$ vs. $2.5 \%, P=0.314)$. Conclusion. $100 \mathrm{mg}$ flurbiprofen axetil can reduce the NGAL at 6 hours after TURP, and it may have a protective effect on the kidney.
\end{abstract}

\section{Introduction}

Acute kidney injury (AKI) is a clinical syndrome characterized by a sharp deterioration in renal function [1]. AKI is one of the serious complications after surgery, and the incidence of perioperative AKI is $6.3 \% \sim 7.4 \%[2,3]$. The occurrence of AKI is related to factors such as perioperative hemodynamic changes, systemic inflammatory response, renal ischemia-reperfusion injury, and oxidative stress [4]. Besides, hypovolemia, dehydration, hypoxia, antibiotics, contrast agents, nonsteroidal drugs, antitumor drugs, and antiretroviral drugs are also risk factors for perioperative
AKI [5]. Perioperative AKI can develop into chronic kidney disease (CKD) and end-stage renal disease (ESRD) and even lead to death [6]. Therefore, the prevention and treatment of perioperative AKI are particularly important.

KDIGO generally uses serum creatinine (Scr) or urine volume changes as diagnostic criteria for the diagnosis of AKI, but these two indicators have limitations and lag. In recent years, many studies have found that some biomarkers can predict the occurrence of AKI earlier than Scr, including CystatinC, NGAL, and N-acetyl- $\beta$-D-glucosaminidase (NAG) [7].

Flurbiprofen axetil is a nonselective cyclooxygenase (COX) inhibitor, which can gather at the surgical incision 
and inhibit the inflammatory response, thereby reducing postoperative pain, so it is widely used in the perioperative period [8]. However, studies have shown that the use of flurbiprofen may cause renal papillary necrosis and tubulointerstitial inflammation $[9,10]$. Studies have shown that nonsteroidal anti-inflammatory drugs (NSAIDs) inhibit the expansion of preglomerular arterioles mediated by prostaglandins by inhibiting COX enzymes, resulting in a decrease in renal blood flow. When renal blood flow is reduced or renal disease is already present, it may cause renal ischemia or acute tubular necrosis (ATN) [11]. Another research has found that NSAIDs can cause hyporeninemia and hyperaldosteronism and can cause ATN, acute tubular interstitial nephritis, glomerulonephritis, renal papillary necrosis, and other nephrotoxicities [12]. Moreover, ATN is an important cause of AKI, and about $40 \%$ of AKI is produced by ATN [13]. It has also been found that NSAIDs can cause acute tubulointerstitial nephritis (ATIN), which may be related to immune response [14]. Although the above studies have shown that NSAIDs are toxic to the kidneys, their wide application in the perioperative period is not limited. The retrospective analysis of our team also found that the effect of flurbiprofen axetil on postoperative renal function is dosedependent in the perioperative period: $50-100 \mathrm{mg}$ flurbiprofen axetil can reduce the incidence of AKI postoperatively, but there is obvious renal damage when it exceeds $250 \mathrm{mg}$ [15]. To this end, we conducted a prospective study to verify whether low-dose flurbiprofen axetil has a protective effect on the kidney by detecting changes in Scr, Cystatin-C, NGAL, and other indicators during the perioperative period.

\section{Method}

In accordance with the CONSORT statement, the present randomized controlled trial was approved by the appropriate Institutional Review Board (IRB) of the Third Xiangya Hospital of Central South University (IRB: R20255), and written informed consent was provided by all patients.

This is a randomized, double-blind, placebo-controlled clinical study. Between August 2020 and November 2020, male patients undergoing TURP under spinal anesthesia at the Third Xiangya Hospital of Central South University were selected. The researcher collected and recorded basic patient information one day before surgery, including age, body mass index (BMI), comorbid diseases, medication history, and test results. Those who met the following criteria were selected: (1) age 60-85 years; (2) ASA classification 1 to 3; (3) $\mathrm{BMI}>18 \mathrm{~kg} / \mathrm{m}^{2}$ and $<30 \mathrm{~kg} / \mathrm{m}^{2}$; (4) patients or their family members could provide written informed consent. Patients were excluded if they met one of the following situations: (1) long-term use of NSAIDs before surgery; (2) flurbiprofen axetil contraindication; (3) severe diseases such as sepsis, severe liver insufficiency (Child-Pugh Grade C), CKD stage $3\left(\mathrm{eGFR} \leq 60 \mathrm{~mL} / \mathrm{min} / 1.73 \mathrm{~m}^{2}\right)$ or higher, NYHA grade 3 to 4 , and moderate or higher anemia $(\mathrm{Hb}<90 \mathrm{~g} / \mathrm{L})$; (4) perioperative use other types of NSAIDs or drugs that affect renal function, including aminoglycoside antibiotics, iodinating agents, contrast agents, antitumor drugs, and antiretroviral drugs; (5) intraoperative hypotension (blood pressure lower than $90 / 60 \mathrm{mmHg}$ or $\mathrm{MAP}<60 \mathrm{mmHg}$ ) continued to exceed $20 \mathrm{~min}$; (6) entered ICU after operation; (7) switched to general anesthesia. The included patients were randomly divided into an experimental group (group F) and a control group (group C) at a ratio of $1: 1$.

2.1. Randomization and Masking. The researchers used SAS statistical software to generate 1:1 random numbers for the experimental group and the control group on the computer to determine the grouping situation. Surgeons, anesthesiologists, researchers, statistical analysts, and patients did not know the study group. Because the flurbiprofen axetil is milky white, the control group $0.9 \%$ normal saline is colorless and transparent. The administer was a nonblind person who was only responsible for the administration and did not participate in information collection, anesthesia, surgery, and statistical analysis.

2.2. Anesthesia and Surgery. After the patient entered the operating room, low-flow oxygen inhalation was performed and vital signs such as blood pressure, electrocardiogram, oxygen saturation, and pulse were monitored. Under the guidance of the anesthesiologist, the patient took the left or right decubitus position, held the knees with both hands, and kept the thighs close to the abdominal wall and the head close to the chest, so that the waist and back were bent back. The anesthesiologist disinfected the towel and used $2 \%$ lidocaine $3 \mathrm{~mL}$ for local anesthesia. Then, he punctured the subarachnoid space in the L3 4 space, through the skin, subcutaneous tissue, supraspinous ligament, interspinous ligament, ligamentum flavum, dura mater, and arachnoid membrane to subarachnoid space, and injected a mixed solution of $0.5 \mathrm{~mL} 10 \%$ glucose solution, $0.025 \mathrm{mg}$ fentanyl, and $1.5 \mathrm{~mL} 1 \%$ ropivacaine hydrochloride. After successful anesthesia, the same group of surgeons performed TURP on the patient. Closely monitored vital signs after the operation and recorded ASA classification, anesthesia plane, infusion, blood loss, operation time, and blood pressure change. After the operation, the two groups of patients were treated with routine treatment and nursing such as oxygen inhalation, ECG monitoring, continuous bladder irrigation, early enteral nutrition, anti-inflammatory, and stomach protection. Meanwhile, flurbiprofen axetil-related complications and adverse reactions should be taken seriously.

2.3. Intervention. 10 minutes before the operation, the patient in group $\mathrm{F}$ received a single injection of $100 \mathrm{mg}(10 \mathrm{~mL})$ flurbiprofen axetil through a peripheral vein, and group $\mathrm{C}$ received a single intravenous injection of the same volume $(10 \mathrm{~mL})$ of $0.9 \%$ normal saline by the same administrator.

\subsection{Outcome Measurement}

2.4.1. Main Outcome. There is an incidence of AKI and eGFR. According to the latest Kidney Disease Improving Global Outcomes (KDIGO) diagnostic criteria for AKI, AKI can be diagnosed if one of the following conditions was met: (1) Scr increased by more than $0.3 \mathrm{mg} / \mathrm{dL}$ $(\geq 26.5 \mu \mathrm{mol} / \mathrm{L}) 48$ hours after surgery compared with the preoperative baseline value; (2) Scr increased by more than 
1.5 times 7 days after operation compared with the baseline level before operation; (3) the hourly urine output is less than $0.5 \mathrm{~mL} / \mathrm{kg}$ and lasts for more than 6 hours [16]. eGFR is calculated by the formula of Chronic Kidney Disease Epidemiology Collaboration (CKD-EPI) [17]: $\mathrm{eGFR}=141 *(\mathrm{Scr} / 0.9) a * 0.993$ year (year represents age. $a$ is a fixed constant, when Scr $>0.9 \mathrm{mg} / \mathrm{dL}, a=-1.209$; when $\mathrm{Scr} \leq 0.9 \mathrm{mg} / \mathrm{dL}, a=-0.411)$. Scr is determined by the Jaffe detection method.

2.4.2. Secondary Outcome. The changes of serum Cystatin-C, NGAL, IL-6, CRP, and other indicators were compared between the two groups of patients at 4 time points (before operation, 6 hours, 24 hours, and 48 hours after the operation). The immunoturbidimetric method was used to determine the contents of Cystatin-C, NGAL, and CRP. Flow cytometry was used to detect the changes of serum IL-6 over time in 12 patients ( 6 in group $\mathrm{F}$ and 6 in group $\mathrm{C}$ ).

\subsection{Drugs and Experimental Reagents}

2.5.1. Flurbiprofen Axetil Injection. The production batch number is H20183054, produced by Wuhan Daan Pharmaceutical Co., Ltd. 0.9\% sodium chloride injection: the production batch number is H43020454, produced by Hunan Kelun Pharmaceutical Co., Ltd. 10\% glucose injection: the production batch number is H20102904, produced by Hunan Kelun Pharmaceutical Co., Ltd. Fentanyl citrate injection: the production batch number is 91D06021, produced by Yichang Renfu Pharmaceutical Co., Ltd. Ropivacaine hydrochloride: the production batch number is NBEM, produced by AstraZeneca Pharmaceuticals, Sweden. NGAL assay kit: the production batch number is 20152401008, produced by Beijing Danda Biotechnology Co., Ltd. Cystatin-C determination kit: the production batch number is 20162400234, produced by Anhui Iprokang Biotechnology Co., Ltd. CRP determination kit: Zhejiang Quark Biotechnology Co., Ltd., medical device registration number: Zhejiang Machinery Standard Note 20192400387. IL-6 kit: the production batch number is 20180531, produced by Agilent Bio (Hangzhou) Co., Ltd.

2.6. Statistical Analysis. SPSS software version 26.0 was used for analysis. Measurement data were expressed in the form of mean \pm standard deviation (mean $\pm \mathrm{SD}$ ) or median (interquartile range). The count data were expressed in percentage (\%). The method of kurtosis and skewness coefficient was used to test the normality of measurement data. The measurement data conforming to the normal distribution adopts the $t$-test, and the measurement data not conforming to the normal distribution adopts the Wilcoxon rank-sum test; the count data adopts the $\chi^{2}$ test or Fisher's exact test. The changes of serum Cystatin-C and NGAL over time and group were analyzed by two-factor repeated-measures analysis of variance. The significance level for all tests was set at $P<0.05$.

\section{Result}

3.1. Subject Recruitment. From August 2020 to November 2020, a total of 88 patients underwent TURP surgery. Among them, 2 patients were younger than 60 years old, 2 patients refused to participate, 2 patients switched to general anesthesia, and 1 patient had a preoperative eGFR less than $60 \mathrm{~mL} / \mathrm{min} / 1.73 \mathrm{~m}^{2} ; 1$ patient used other types of NSAIDs perioperatively. In the end, 80 patients were enrolled, 40 in group $\mathrm{F}$ and 40 in group $\mathrm{C}$ (Figure 1).

3.2. Baseline of the Two Groups. As shown in Table 1, there were no statistically significant differences in age, BMI, ASA classification, preoperative mean arterial pressure, preoperative comorbidities, level of anesthesia, operation time, and infusion volume between the two groups. From the perspective of preoperative medication, the preoperative dosage of angiotensin-converting enzyme inhibitor (ACEI), angiotensin receptor blockers (ARB), or calcium channel blockers (CCB) was not significantly different between the two groups of patients.

3.3. Comparison of Lab Results between the Two Groups. The Scr, eGFR, and hemoglobin before the operation and 48 hours after operation were no significant difference between the two groups. The CRP in group $\mathrm{F}$ was lower than that in group $\mathrm{C}$ at 48 hours after surgery $(P=0.005)$, but there was no significant difference preoperatively (Table 2 ). Table 3 shows the changes in serum IL- 6 of 12 patients over time ( 6 in group C and 6 in group F). The level of serum IL- 6 in group $\mathrm{C}$ was significantly higher than that in group $\mathrm{F}$ at 6 hours after the operation $(P=0.004)$.

3.4. The Incidence of AKI in the Two Groups. A total of 80 patients were included in the analysis. One patient in group $\mathrm{C}$ developed AKI, and the incidence was $2.5 \%$. There was no AKI patient in group $\mathrm{F}$, and there was no significant difference in the incidence of AKI between the two groups $(P=0.314)$. Also, there was no significant difference in blood pressure between the two groups of patients from entering the operating room to leaving the operating room $(P>0.05)$.

3.5. Changes in Biomarkers. The effects of group and time on Cystatin- $\mathrm{C}$ are as follows:

The interaction between group and time had no significant difference on Cystatin-C $(P=0.109)$. There was no statistical difference in Cystatin- $\mathrm{C}$ between group $\mathrm{F}$ and group $\mathrm{C}$ at the same time point $(P=0.913)$. The Cystatin- $C$ were compared at different time points in the two groups, and it was found that Cystatin-C decreased with time from 0 to 6 hours after surgery $(P<0.001)$, but it began to rise again from 24 hours to 48 hours after surgery $(P<0.001)$. The changes of Cystatin- $\mathrm{C}$ in the two groups with time are shown in Figure 2.

The effects of group and time on serum NGAL levels are as follows:

The interaction between group and time had a significant difference on NGAL $(P=0.002)$. At 6 hours after the operation, the NGAL level in group F was significantly lower than that in group $\mathrm{C}(P=0.002)$. However, there was no significant difference in NGAL between the two groups before the operation, $24 \mathrm{~h}$ after operation, and $48 \mathrm{~h}$ after operation $(P>0.05)$. NGAL were compared at different time points in the two groups: in group C, NGAL showed an upward trend 


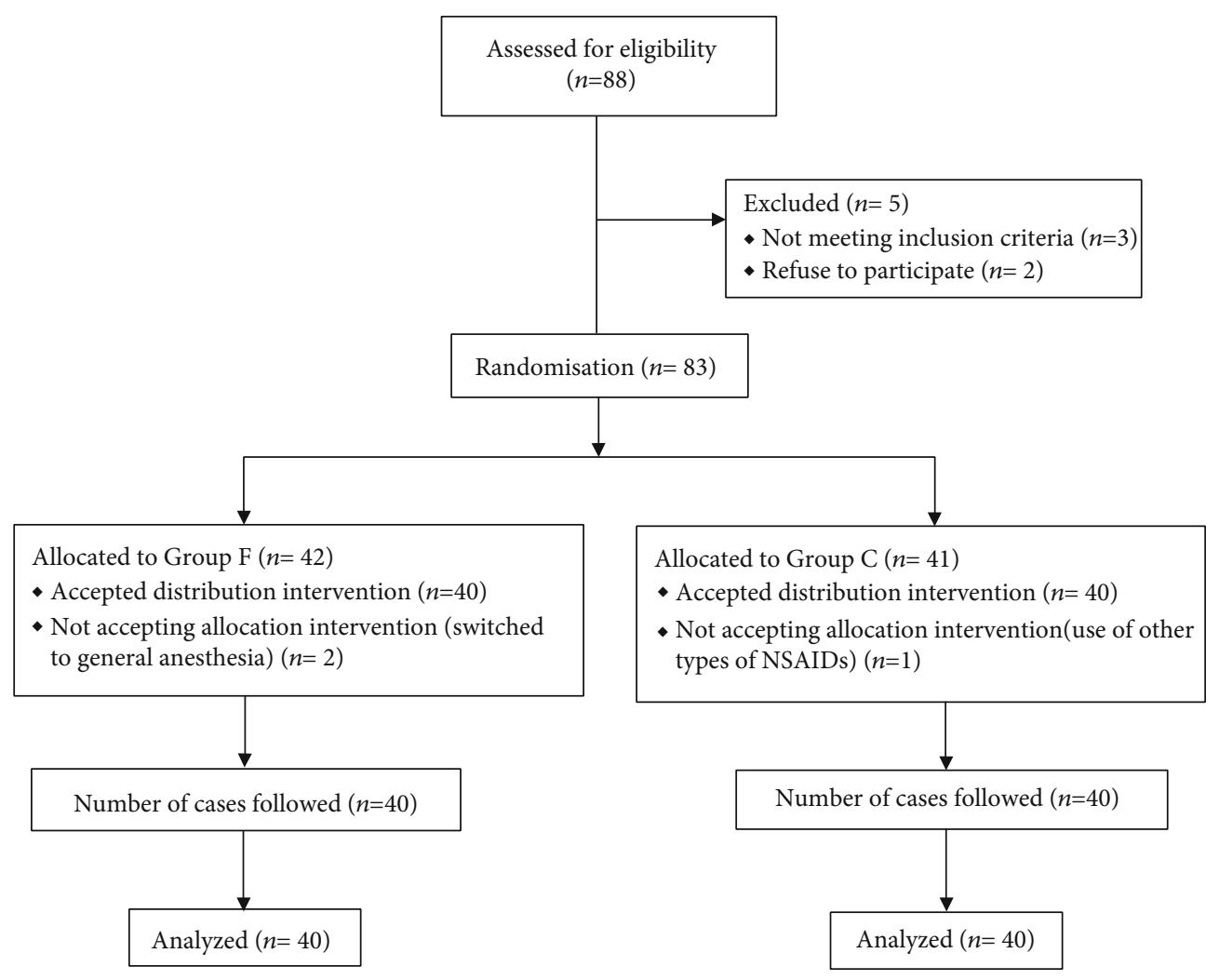

Figure 1: Subject recruitment.

TABLE 1: Comparison of the basic characteristics of the two groups of patients.

\begin{tabular}{lcc}
\hline & Group C $(n=40)$ & Group F $(n=40)$ \\
\hline Mean \pm SD age $($ year $)$ & $70.68 \pm 7.70$ & $68.40 \pm 8.14$ \\
Mean \pm SD BMI $\left(\mathrm{kg} / \mathrm{m}^{2}\right)$ & $22.90 \pm 3.28$ & $23.02 \pm 3.09$ \\
Mean \pm SD preoperative MAP $(\mathrm{mmHg})$ & $95.56 \pm 9.19$ & $98.75 \pm 11.42$ \\
Preoperative comorbidities & & $12(30)$ \\
High blood pressure & $14(35)$ & $3(7.5)$ \\
Diabetes & $7(17.5)$ & 0.872 \\
Preoperative medication & & $1(0)$ \\
ACEI & $2(2.5)$ & $9(22.5)$ \\
ARB & $2(2.5)$ & 0.172 \\
CCB & $10(25)$ & $3(7.5)$ \\
Anesthesia plane & & $20(50)$ \\
T6 S5 & $0(0)$ & $17(42.5)$ \\
T8 S5 & $17(42.5)$ & 0.176 \\
T10 S5 & $23(57.0)$ & 0.152 \\
Mean \pm SD operation time $(\mathrm{min})$ & $69.93 \pm 20.87$ & 0.793 \\
Mean \pm SD infusion volume $(\mathrm{mL})$ & $701.50 \pm 205.78$ & $736.50 \pm 186.64$ \\
\hline
\end{tabular}

Data are number of patients (\%) unless otherwise indicated. BMI: body mass index; MAP: mean arterial pressure; ACEI: angiotensin-converting enzyme inhibitor; ARB: angiotensin receptor blockers; CCB: calcium channel blockers.

within 6 hours after surgery $(P=0.010)$, but there was no significant difference from 6 to 48 hours after surgery $(P>0.05)$; in group F, the effect of time on NGAL was no significant dif- ference, that is, there was no significant difference in NGAL with time $(P>0.05)$. The changes of NGAL in the two groups over time are shown in Figure 3. 
TABLE 2: Comparison of lab results between two groups of patients.

\begin{tabular}{lccc}
\hline & Group C $(n=40)$ & Group F $(n=40)$ & $P$ value \\
\hline Before surgery & & & \\
Scr $(\mu \mathrm{mol} / \mathrm{L})$ & $81.40 \pm 13.11$ & $80.70 \pm 12.67$ & 0.806 \\
eGFR $\left(\mathrm{mL} / \mathrm{min} / 1.73 \mathrm{~m}^{2}\right)$ & $84.59 \pm 17.81$ & $85.14 \pm 15.83$ & 0.883 \\
$\mathrm{Hb}(\mathrm{g} / \mathrm{L})$ & $133.18 \pm 13.52$ & $134.91 \pm 17.71$ & 0.624 \\
$\mathrm{CRP}(\mathrm{mg} / \mathrm{L})$ & $1.76 \pm 1.92$ & $1.93 \pm 1.1$ & 0.562 \\
48 hours after surgery & & & \\
Scr $(\mu \mathrm{mol} / \mathrm{L})$ & $86.63 \pm 16.15$ & $78.43 \pm 16.73$ & 0.819 \\
eGFR $\left(\mathrm{mL} / \mathrm{min} / 1.73 \mathrm{~m}^{2}\right)$ & $79.17 \pm 19.13$ & $134.20 \pm 13.75$ & 0.824 \\
Hb $(\mathrm{g} / \mathrm{L})$ & $132.00 \pm 12.73$ & $10.33 \pm 11.46$ & 0.460 \\
$\mathrm{CRP}(\mathrm{mg} / \mathrm{L})$ & $18.04 \pm 17.00$ & $0.005^{*}$
\end{tabular}

Data are shown as mean \pm SD. Scr: serum creatinine; eGFR: glomerular filtration rate; Hb: hemoglobin; CRP: C reactive protein. * Significant $P$ value.

TABLE 3: The changes of IL-6 in the two groups over time.

\begin{tabular}{lcccc}
\hline & Preoperative & \multicolumn{2}{c}{ Postoperative } & 24 hours \\
\hline Group C $(n=6)$ & $4.10(2.76-8.31)$ & $11.28(8.43-36.40)$ & $11.66(4.97-25.14)$ & $7.94(4.84-23.00)$ \\
Group F $(n=6)$ & $3.17(2.38-4.32)$ & $3.16(2.38-3.77)$ & $3.92(2.84-12.66)$ & $3.36(2.49-16.43)$ \\
$P$ value & 0.262 & $0.004^{*}$ & 0.092 & 0.109 \\
\hline
\end{tabular}

Data are shown as median (IQR). * Significant $P$ value.

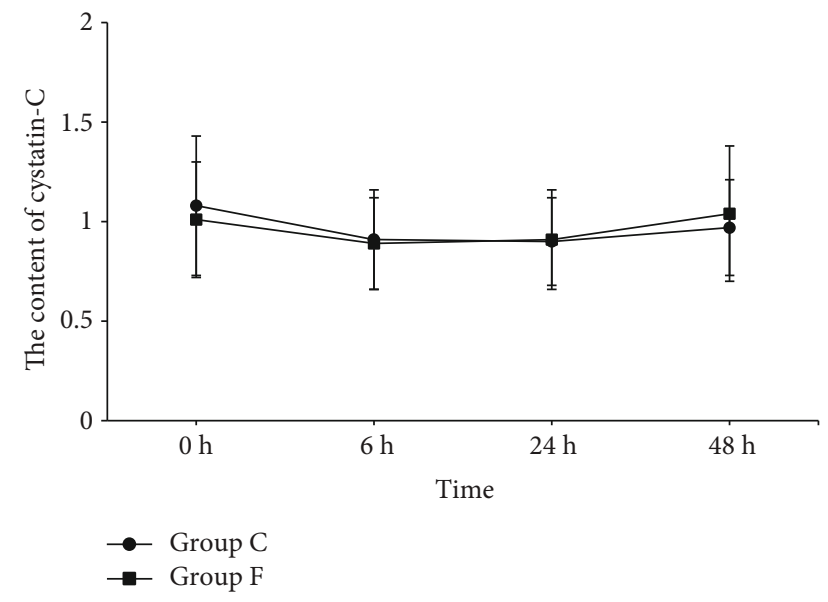

Figure 2: The changes of Cystatin-C in the two groups over time $0 \mathrm{~h}, 6 \mathrm{~h}, 24 \mathrm{~h}$, and $48 \mathrm{~h}$ represent preoperatively, 6 hours, 24 hours, and 48 hours postoperatively, respectively.

\section{Discussion}

In this study, only 1 patient developed AKI (1.3\%) after TURP, which was similar to the extremely rare incidence of AKI after TURP showed in previous studies [18]. However, some studies believed that old age, past diseases, and urinary tract obstruction are high-risk factors for postoperative renal injury $[19,20]$. For TURP, hemolytic reactions that occur in the bladder and surgical wounds were infused with sterile saline during the operation. The hemoglobin produced by this reaction could also cause renal tubular damage after

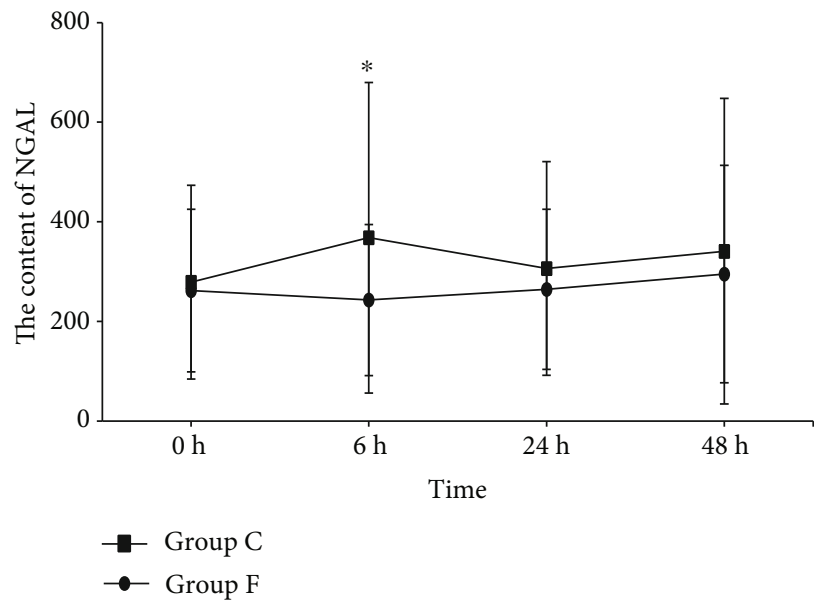

FIgURE 3: The changes of NGAL in the two groups over time $0 \mathrm{~h}$, $6 \mathrm{~h}, 24 \mathrm{~h}$, and $48 \mathrm{~h}$ represent preoperatively, 6 hours, 24 hours, and 48 hours postoperatively, respectively. ${ }^{*} P<0.05$.

being absorbed by the bladder and blood [21, 22]. Therefore, our study concluded that in the control group, the NGAL concentration at 6 hours after surgery was significantly higher than that before surgery, indicating that although surgery-related AKI caused by TURP was rare, renal tubular damage caused by surgery can still be observed.

In the experimental group, with a small dose of flurbiprofen axetil, the serum NGAL concentration at 6 hours after surgery did not increase compared with that before the operation, which suggested that flurbiprofen axetil may be related to the protection of renal tubules [7]. As early as 1991, it was 
reported that the application of flurbiprofen axetil can cause renal papillary necrosis and renal tubulointerstitial inflammation $[9,10]$. As we all know, NSAIDs can inhibit COX enzymes and reduce renal blood flow by blocking prostaglandin-mediated dilation of the glomerular arterioles. The decline of renal blood flow can cause renal ischemia to be further aggravated or renal tubular necrosis in the case of existing renal disease [11]. In a single-center retrospective study, it was found that compared with patients who did not use flurbiprofen axetil, patients who received $100 \mathrm{mg}$ flurbiprofen axetil during the perioperative period had a lower incidence of AKI [15]. These studies have suggested that flurbiprofen axetil may have renal protection.

Although the mechanism of action of flurbiprofen axetil in protecting renal tubules is still unclear, the release of inflammatory mediators is the basis of renal tubular damage [23]. Takaku et al. found that the use of COX-2 enzyme inhibitors can reduce the levels of systemic inflammatory mediators (such as IL- $1 \alpha$, IL- $1 \beta$, IL-6, and TNF- $\alpha$ ) and kidney damage in shock mouse models [24]. In this study, the level of IL- 6 in the experimental group was significantly lower than that in the control group at 6 hours after surgery, similar to the results of the former study, suggesting that flurbiprofen axetil can reduce the level of inflammation in the body and reduce renal tubular damage. In addition, the study by Feitoza et al. showed that before renal ischemia and reperfusion, the use of nonselective COX enzyme inhibitors reduces the development of renal fibrosis through its antiinflammatory effects [25]. In this study, the CRP of the control group was significantly higher than that of the experimental group at 48 hours after the operation due to the inhibitory effect of the COX-2 enzyme $(P=0.005)$. It suggests that compared with the control group, the inflammatory response of the experimental group was significantly lower than that of the control group. It can be seen that flurbiprofen axetil may produce renal tubular protection through its anti-inflammatory mechanism.

In addition, in the case of renal hypoperfusion, the increased expression of COX-2 in the dense macula contributes to the renal vasoconstriction response, and the renal blood flow and glomerular filtration rate decrease through the tubule-glomerular feedback mechanism [26, 27]. It is speculated that, as a nonselective COX enzyme inhibitor, flurbiprofen axetil can also inhibit the COX enzyme in the dense macula of the kidney, thereby improving renal blood flow and protecting renal function. Finally, the COX-2 enzyme can lead to the synthesis and secretion of transforming growth factor (TGF- $\beta$ ), which in turn leads to tubular deposition of collagen and fibrin in the kidney [28]. Cheng and Harris believe that long-term use of selective COX-2 inhibitors shows potential renal protection in cases of glomerular hyperfiltration, such as diabetes and partial nephrectomy, and reduces proteinuria, extracellular matrix deposition, and glomerulosclerosis [29].

Due to various reasons, this research still has some limits. First of all, this was a single-center prospective study. Whether flurbiprofen axetil has a protective effect during the perioperative period requires clinical verification in multiple centers and multiple populations. Second, due to the short hospital stay of patients undergoing transurethral prostate surgery and continuous bladder lavage after the operation, data on postoperative urine output and Scr 7 days after surgery were not available, so only the changes in renal function after 48 hours could be observed. The actual incidence of AKI during the perioperative period might be underestimated. Third, in addition to the detection of IL-6, this study did not observe other perioperative inflammatory mediators (such as IL- $1 \alpha$, IL- $1 \beta$, and TNF- $\alpha$ ). The mechanism of the renal protection of flurbiprofen axetil may need to be verified by animal experiments.

\section{Conclusion}

$100 \mathrm{mg}$ flurbiprofen axetil can reduce the NGAL at 6 hours after TURP and may have a protective effect on the kidneys.

\section{Data Availability}

The data used to support the findings of this study may be released upon application to the Department of Anesthesiology, Third Xiangya Hospital, Central South University, which can be contacted at xy3irb@163.com

\section{Conflicts of Interest}

The authors declare that they have no competing interests.

\section{Authors' Contributions}

Dong Wang and Wenxiu Xie contributed equally to this work.

\section{Acknowledgments}

This work was supported by the Project of Health and Health Commission of Hunan Province (20201802), Hunan Province Key Laboratory Project (2018TP1009), Natural Science Foundation of Hunan Province (2020JJ5854), National Natural Science Foundation of China (81901842), China Primary Health Care Foundation (YLGX-WS2020003), and Research and Innovation Funds from Xiangya Bigdata Foundation of Central South University.

\section{References}

[1] R. Bellomo, J. A. Kellum, and C. J. L. Ronco, "Acute kidney injury," Lancet, vol. 380, no. 9843, pp. 756-766, 2012.

[2] M. Walsh, P. J. Devereaux, A. X. Garg et al., "Relationship between intraoperative mean arterial pressure and clinical outcomes after noncardiac surgery: toward an empirical definition of hypotension," Anesthesiology, vol. 119, no. 3, pp. 507-515, 2013.

[3] L. Y. Sun, D. N. Wijeysundera, G. A. Tait, and W. S. Beattie, "Association of intraoperative hypotension with acute kidney injury after elective noncardiac surgery," Anesthesiology, vol. 123, no. 3, pp. 515-523, 2015.

[4] J. Vanmassenhove, J. Kielstein, A. Jörres, and W. V. Biesen, "Management of patients at risk of acute kidney injury," Lancet, vol. 389, no. 10084, pp. 2139-2151, 2017.

[5] R. L. Mehta, J. Cerdá, E. A. Burdmann et al., "International Society of Nephrology's 0 by 25 initiative for acute kidney injury 
(zero preventable deaths by 2025): a human rights case for nephrology," Lancet, vol. 385, no. 9987, pp. 2616-2643, 2015.

[6] M. E. Grams, Y. Sang, J. Coresh et al., “Acute kidney injury after major surgery: a retrospective analysis of veterans health administration data," American Journal of Kidney Diseases, vol. 67, no. 6, pp. 872-880, 2016.

[7] M. Ostermann, "Acute kidney injury 2016: diagnosis and diagnostic workup," Critical Care, vol. 20, no. 1, p. 299, 2016.

[8] K. Wang, J. Luo, L. Zheng, and T. Luo, "Preoperative flurbiprofen axetil administration for acute postoperative pain: a meta-analysis of randomized controlled trials," Journal of Anesthesia, vol. 31, no. 6, pp. 852-860, 2017.

[9] J. Kaufhold and M. Wilkowski, "Flurbiprofen-associated acute tubulointerstitial nephritis," American Journal of Nephrology, vol. 11, no. 2, pp. 144-146, 1991.

[10] H. Berning, K. Orellana, and W. Selberg, "Nierenpapillennekrosen," DMW - Deutsche Medizinische Wochenschrift, vol. 99, no. 36, pp. 1749-1754, 1974.

[11] S. Clavé, C. Rousset-Rouvière, L. Daniel, and M. Tsimaratos, "The invisible threat of non-steroidal anti-inflammatory drugs for kidneys," Frontiers in Pediatric, vol. 7, 2019.

[12] A. Nessa, "Nonsteroidal anti-inflammatory drugs (NSAIDS) induced acute kidney injury (AKI): patient profile and outcome in Bangladesh armed forces," JAFMC, vol. 10, no. 2, pp. 39-43, 2015.

[13] Y. Nechemia-Arbely, D. Barkan, G. Pizov et al., "IL-6/IL-6R axis plays a critical role in acute kidney injury," Journal of the American Society of Nephrology, vol. 19, no. 6, pp. 11061115, 2008.

[14] J. J. K. I. Rossert, "Drug-induced acute interstitial nephritis," Kidney International, vol. 60, no. 2, pp. 804-817, 2005.

[15] D. Wang, S. K. Yang, M. X. Zhao et al., "Low dose of flurbiprofen axetil decrease the rate of acute kidney injury after operation: a retrospective clinical data analysis of 9915 cases," BMC Nephrology, vol. 21, no. 1, p. 52, 2020.

[16] P. A. J. N. C. Khwaja, "KDIGO clinical practice guidelines for acute kidney injury," Nephron. Clinical Practice, vol. 120, no. 4, pp. c179-c184, 2012.

[17] L. ASJAoim, "A new equation to estimate glomerular filtration rate," Annals of Internal Medicine, vol. 150, no. 9, pp. 604-612, 2009.

[18] E. C. Costalonga, "Prostatic surgery associated acute kidney injury," World Journal of Nephrology, vol. 3, no. 4, pp. 98209, 2014.

[19] C. Y. Bilen, A. Şahin, H. Özen, F. T. Aki, Ö. Öge, and S. Kendi, "Nonoliguric renal failure after transurethral resection of prostate," Journal of Endourology, vol. 13, no. 10, pp. 751-754, 1999.

[20] D. P. Shipstone, "Irrigating fluids in endoscopic surgery," BJU International, vol. 79, no. 5, pp. 669-680, 1997.

[21] G. Orłowska-Kowalik, L. Janicka, and A. J. W. L. Ksiazek, "Acute non-inflammatory renal failure after transurethral electroresection of the prostate combined with irrigation of the bladder with distilled water," Wiadomości Lekarskie, vol. 42, no. 9, pp. 608-611, 1989.

[22] R. A. Zager, "Pathogenetic mechanisms in experimental hemoglobinuric acute renal failure," American Journal of PhysiologyRenal Physiology, vol. 256, no. 3, pp. F446-F455, 1989.

[23] J. V. Bonventre and A. J. K. I. Zuk, "Ischemic acute renal failure: an inflammatory disease?," Kidney International, vol. 66, no. 2, pp. 480-485, 2004.
[24] M. Takaku, A. C. da Silva, N. I. Iritsu, P. T. G. Vianna, and Y. M. M. Castiglia, "Effects of a single dose of parecoxib on inflammatory response and ischemic tubular injury caused by hemorrhagic shock in rats," Pain Research and Treatment, vol. 2018, Article ID 8375746, 8 pages, 2018.

[25] C. Q. Feitoza, G. M. Gonçalves, P. Semedo et al., "Inhibition of COX 1 and 2 prior to renal ischemia/reperfusion injury decreases the development of fibrosis," Molecular Medicine, vol. 14 , no. $11-12$, pp. 724-730, 2008.

[26] M. Brezis and S. Rosen, "Hypoxia of the renal medulla - its implications for disease," New England Journal of Medicine, vol. 332, no. 10, pp. 647-655, 1995.

[27] M. Araujo and W. J. Welch, "Cyclooxygenase 2 inhibition suppresses tubuloglomerular feedback: roles of thromboxane receptors and nitric oxide," American Journal of PhysiologyRenal Physiology, vol. 296, no. 4, pp. F790-F794, 2009.

[28] A. Miyajima, K. Ito, T. Asano, K. Seta, A. Ueda, and M. Hayakawa, "Does cyclooxygenase-2 inhibitor prevent renal tissue damage in unilateral ureteral obstruction?," Journal of Urology, vol. 166, no. 3, pp. 1124-1129, 2001.

[29] H. F. Cheng and R. C. J. H. Harris, "Cyclooxygenases, the kidney, and hypertension," Hypertension, vol. 43, no. 3, pp. 525530, 2004. 